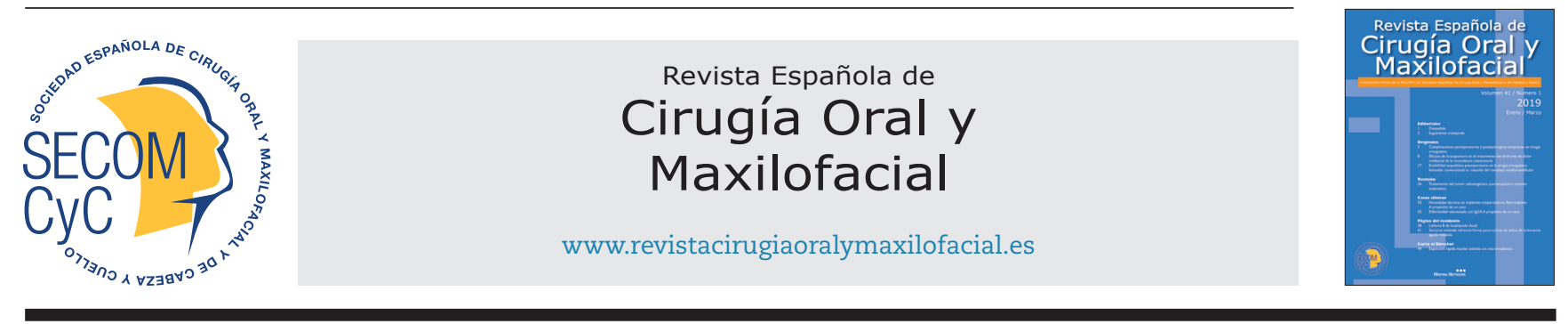

\title{
Revisión
}

\section{Profilaxis antibiótica en implantología oral. Revisión crítica de la literatura}

\author{
Ángel-Orión Salgado-Peralvoㄹ, Javier Sanz-Esporrín², María-Victoria Mateos-Moreno3, \\ Ahmad Haidar-Wehbe ${ }^{4}$, Andrés Blanco-Carrión ${ }^{5}$ y Eugenio Velasco-Ortega ${ }^{6}$
}

\begin{abstract}
${ }^{1}$ Magister en Implantología Oral. Magister en Odontología Familiar y Comunitaria. Universidad de Sevilla. Práctica privada. Vigo. ${ }^{2}$ Magister en Periodoncia e Implantes. Magíster en Ciencias Odontológicas. Universidad Complutense de Madrid. ${ }^{3}$ Profesora asociada, Departamento de Especialidades Clínicas Odontológicas, Facultad de Odontología, Universidad Complutense de Madrid. ${ }^{4}$ Magister en Implantología Oral, Universidad de Sevilla. Magister en Ciencias Odontológicas, Universidad Complutense de Madrid. ${ }^{5}$ Profesor titular de Medicina Oral. Director del Máster Odontología Práctica Diaria, Universidad de Santiago de Compostela. ${ }^{6}$ Profesor titular de Odontología Integrada de Adultos y Gerodontología. Director del Máster en Implantología Oral, Universidad de Sevilla. España
\end{abstract}

\section{INFORMACIÓN DEL ARTÍCULO}

Historia del artículo:

Recibido el 11 de noviembre de 2018 Aceptado el 15 de febrero de 2019

Palabras clave:

Implantes dentales, complicaciones de implantes dentales, fracaso de implantes dentales, profilaxis antibiótica.

\section{R E S U M E N}

\begin{abstract}
La masiva difusión de los tratamientos implantológicos conlleva, según los patrones de prescripción actuales, la administración de antibióticos profilácticos, los cuales no están indicados en la mayoría de los casos; sin embargo, su uso suele basarse en la experiencia del operador, en muchos casos influenciado por presiones legales. El desarrollo creciente a nivel mundial de resistencias bacterianas a la casi totalidad de familias de antibióticos conocidos provoca que cada vez sea mayor el número de infecciones cuyo tratamiento se vuelve más complicado debido a la pérdida de eficacia de estos fármacos, lo que constituye un problema fundamental de salud pública.

La evidencia disponible respecto a la prescripción profiláctica antibiótica en tratamientos de implantes muestra que la administración de 2 gramos de amoxicilina, 1 hora antes de la intervención, disminuye significativamente el riesgo de fracaso temprano, no así el riesgo de infección. Pese a ello, no está justificado su uso indiscriminado, sino que se debería prescribir en pacientes en riesgo de sufrir una endocarditis bacteriana, en pacientes inmunodeprimidos, en la inserción de implantes en alvéolos infectados, en cirugías extensas y prolongadas, y en cirugías regenerativas por un mayor riesgo de dehiscencia mucosa, debido a un mayor riesgo de infección asociada a estos casos. Su prescripción en pacientes sanos, sin condicionantes anatómicos y en lechos quirúrgicos con una correcta calidad ósea no estaría justificado.
\end{abstract}

${ }^{*}$ Autor para correspondencia

Correo electrónico: orionsalgado@hotmail.com (Ángel-Orión Salgado-Peralvo).

DOI: 10.20986/recom.2019.1011/2018

1130-0558/@ 2019 SECOM. Publicado por Inspira Network. Este es un artículo Open Access bajo la licencia CC BY-NC-ND (http:// creativecommons.org/licenses/by-nc-nd/4.0/). 


\section{Antibiotic prophylaxis in oral implantology. A critical review of the literature}

\section{A B S T R A C T}

Keywords:

Dental implants, dental implant complications, dental implant failure, antibiotic prophylaxis.
The high frequency of the implant procedures leads to massive use of prophylactic antibiotic treatments. These prescriptions are not indicated in the majority of the cases, are only based on the experience of the surgeon and influenced by legal reasons. Global increase in bacterial resistance to almost all known antibiotic families cause, not only an increase in the number of infections, but also severe difficulties in their management, which leads to a public health problem.

Available literature regarding prophylactic antibiotic prescriptions for implant treatments shows that the administration of 2gr of amoxicillin 1 hour prior to the surgery reduces significantly early implant failure risk. However, risk of infection is not reduced. Despite this fact, indiscriminated antibiotic use is not justified and it should be restricted to patients that have proven risk of suffering from bacterial endocarditis, immunosuppressed patients, implant insertion in infected alveolous, large surgeries and regenerative surgeries due to the increased potential of mucosal dehiscence and increased risk of infection. Antibiotic prescription in healthy patients without anatomical considerations and with acceptable bone quality is not reasonable.

\section{INTRODUCCIÓN}

La antibioterapia en implantología oral puede clasificarse en profiláctica (con el fin de prevenir infecciones) o terapéutica (como tratamiento de infecciones ya instauradas). Frecuentemente, los dentistas se encuentran ante el dilema de prescribir o no antibióticos de manera profiláctica en tratamientos de implantes dentales, constituyendo en la actualidad un tema controvertido. Se ha aceptado su prescripción para evitar bacteriemias sistémicas ${ }^{1}$, aunque también para alcanzar una concentración de antibiótico adecuada en sangre que prevenga la contaminación bacteriana durante el acto quirúrgico de los implantes o del material injertado ${ }^{2}$, ya que la cavidad oral per se constituye una cavidad séptica. A pesar de ello, la prescripción sistemática de antibioterapia en pacientes sanos no presenta una relación riesgo-beneficio justificada ${ }^{3-5}$. El motivo fundamental es el desarrollo creciente a nivel mundial de resistencias bacterianas a prácticamente la totalidad de familias de antibióticos conocidos, que provoca que cada vez sea mayor el número de infecciones cuyo tratamiento se vuelve más difícil, debido a la pérdida de la eficacia de estos fármacos ${ }^{6}$, además de aumentar la probabilidad de reacciones adversas idiosincrásicas y dosis-dependientes que pueden llegar a comprometer la vida del paciente ${ }^{7}$. En este sentido, se estima que por cada millón de pacientes tratados con una única dosis de antibiótico se producen reacciones alérgicas leves, moderadas y severas en 2400,400 y 0,9 pacientes, respectivamente ${ }^{8}$. Otros problemas relacionados con su consumo son la toxicidad directa, que incluye problemas gastrointestinales (náuseas, vómitos, diarrea y dolor abdominal), hematológicos (neutropenia, trombocitopenia y hemolisis), alteraciones en la flora bacteriana habitual de las mucosas, que puede derivar en infecciones por cándida o colitis pseudomembranosa, nefrotoxicidad (proteinuria o fallo renal), neuropatías (disfunción nerviosa o neuropatía periférica), alteraciones hepatobiliares (ictericia o hepatitis) e interacciones medicamentosas ${ }^{9}$.
Los antibióticos son medicamentos utilizados para prevenir y tratar las infecciones bacterianas, y la resistencia a ellos se produce cuando las bacterias mutan en respuesta a su empleo. Es un fenómeno natural, aunque el uso injustificado de estos fármacos en el ser humano, en los animales destinados a la producción de alimentos y en el medio ambiente está acelerando el proceso. Además, la resistencia a los antibióticos prolonga las estancias hospitalarias, incrementa los costos médicos y aumenta la mortalidad, por lo que constituye un problema fundamental de salud pública. Por ello, es imprescindible que se modifique urgentemente la forma de prescribir y utilizar los antibióticos ya que, aunque se desarrollen nuevos fármacos, si no se cambian las pautas de prescripción actuales, las resistencias seguirán suponiendo una grave amenaza. Si no se toman medidas urgentes, el mundo está abocado a una era posantibiótica, en la que muchas infecciones comunes y lesiones menores volverán a ser potencialmente mortales ${ }^{6}$. En concreto, las resistencias antimicrobianas son causantes de más de 25.000 muertes al año en la Unión Europea $^{10}$. Los dentistas representan una parte importante en la sensibilización ante este problema, ya que se estima que, en España, las infecciones odontológicas representan en torno al $10 \%$ de las prescripciones de antibióticos ${ }^{11,12}$. Particularmente, el $72-85,5 \%$ de los dentistas de Finlandia, India, Suecia, Reino Unido y EE. UU. prescriben rutinariamente antibióticos de manera preoperatoria y/o posoperatoriamente ${ }^{13-18}$.

El objetivo del presente manuscrito es realizar una revisión crítica de la literatura acerca de la necesidad, o no, de administrar profilaxis antibiótica en implantología oral, y establecer recomendaciones clínicas al respecto.

\section{MATERIAL Y MÉTODOS}

Para la realización del presente artículo se llevaron a cabo 4 búsquedas independientes en la base de datos de Medline (vía PubMed) de los últimos 10 años (31 de diciembre de 2008 
a 31 de diciembre de 2018) con los términos MESH (Medical Subjects Headings) siguientes: 1) "antibiotic prophylaxis" AND "dental implant"; 2) "antibiotic" AND “immediate implant"; 3) "infectious endocarditis" AND "dental implant"; y 4) "antibiotic prophylaxis" AND "sinus lift".

A su vez se realizó una búsqueda manual en BUCea (buscador de literatura científica de la Universidad Complutense de Madrid) y en Google Scholar, y se analizaron las referencias bibliográficas de los artículos seleccionados en pos de artículos relacionados con la profilaxis antibiótica en implantología oral bajo determinadas circunstancias (en condiciones normales, en pacientes médicamente comprometidos, implantes inmediatos y en lechos infectados, elevaciones de seno maxilar, cirugías óseas regenerativas y en la prevención de endocarditis bacterianas). Únicamente se incluyeron revisiones sistemáticas y metanálisis de estudios realizados en humanos y publicados en inglés o español.

\section{RESULTADOS}

Dos revisores independientes leyeron los títulos y resúmenes de los 17 artículos resultantes en la búsqueda inicial realizada en Medline (vía PubMed). Tras la eliminación de tres artículos duplicados, ambos autores se leyeron el texto completo de los 13 artículos resultantes, excluyendo dos de ellos por no estar relacionados con la prescripción de antibióticos profilácticos en implantología oral, resultando en 11 artículos incluidos en la presente revisión (Figura 1).

Se realizaron recomendaciones clínicas en cuanto al uso preventivo de estos fármacos en el tratamiento de implantes dentales bajo determinadas circunstancias. Respecto a su administración en pacientes bajo condiciones normales (entendiendo como tal a pacientes sin patologías médicas añadidas e implantes sin condiciones anatómicas reseñables que dificulten la colocación de implantes dentales), en implantes inmediatos y en lechos infectados se tomaron como referencia revisiones sistemáticas y metanálisis (Tabla I). Por otro lado, respecto a su prescripción en pacientes con patologías médicas, en implantes que requieren la realización de elevaciones sinusales, cirugías óseas regenerativas y en pacientes en riesgo de desarrollar endocarditis bacteriana, únicamente se disponen de recomendaciones clínicas, por lo que las conclusiones extraídas deben ser consideradas para casos en concreto.

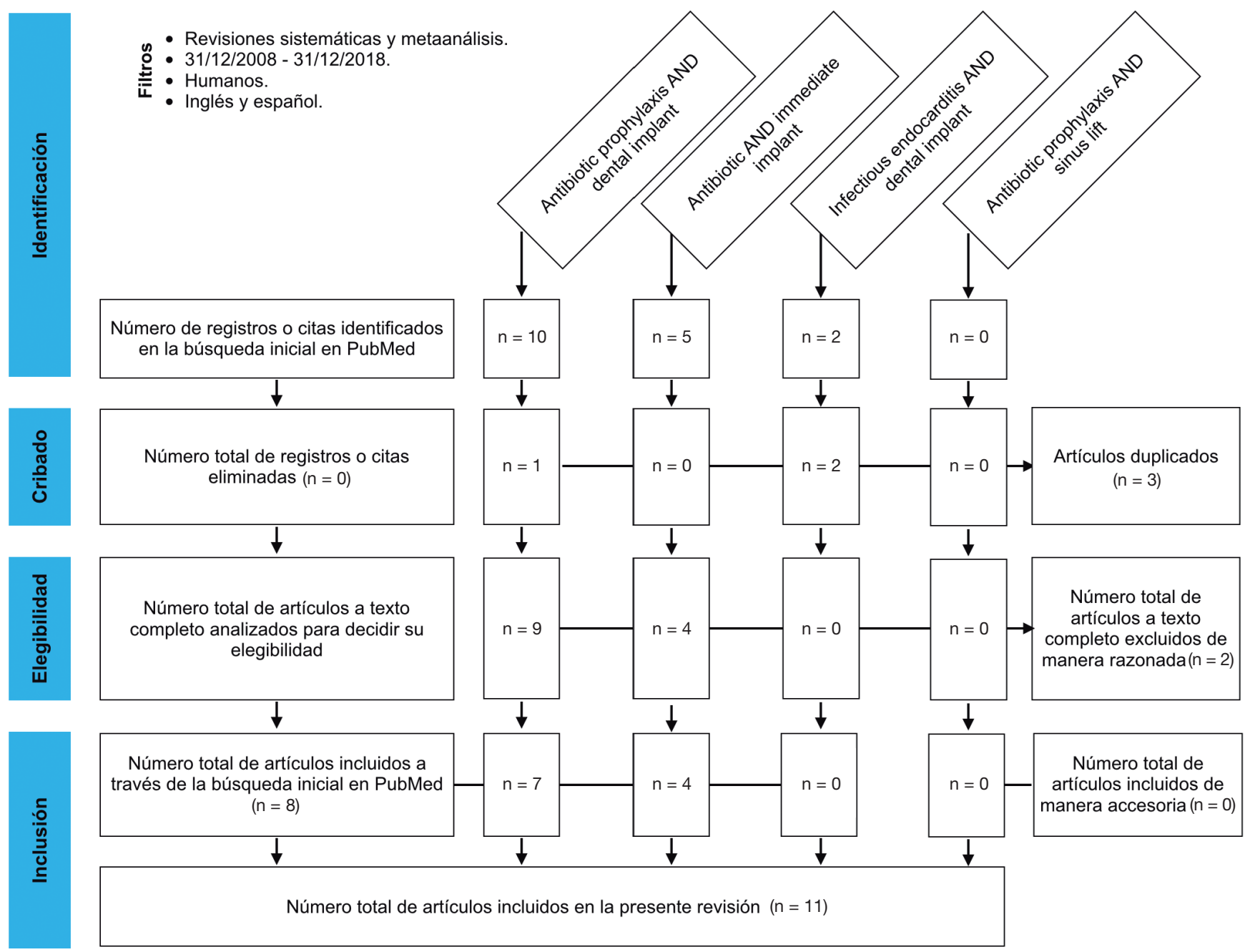

Figura 1. Diagrama de flujo de la búsqueda realizada. 
Tabla I. Relación de artículos analizados en la presente revisión por orden cronológico Autor (es) Tipo de artículo Intervención

Singh-Gill y cols. ${ }^{19}$ (2018)

Revisión sistemática y

metanálisis

Condiciones normales

Rodríguez-Sánchez y

cols. ${ }^{20}$ (2018)

Revisión sistemática y

metanálisis

Condiciones normales

Chen y cols. ${ }^{21}(2017)$

Revisión sistemática

Condiciones normales

Lund y cols. ${ }^{5}(2015)$

Revisión sistemática

Condiciones normales

Chrcanovic y cols. ${ }^{22}$ (2015) Revisión sistemática

Chrcanovic y cols. ${ }^{4}$ (2014) Metanálisis

Ata-Ali y cols. ${ }^{23}$ (2014) Revisión sistemática y metanálisis

Revisión sistemática y metanálisis

Espósito y cols. ${ }^{24}(2013)$

Lang y cols. ${ }^{25}$ (2012)

Revisión sistemática

Waasdorp y cols. ${ }^{26}$ (2010) Revisión sistemática

Espósito y cols.? (2010)

Revisión sistemática y metanálisis
Inserción inmediata de implantes

Inserción de implantes en lechos infectados

Inserción de implantes en lechos infectados

Condiciones normales

Condiciones normales

Condiciones normales

Condiciones normales

\section{Conclusiones}

No existe un beneficio añadido en la prescripción profiláctica de antibióticos en implantología oral en condiciones normales con el fin de reducir las tasas de fracaso temprano e infecciones posoperatorias

La prescripción de amoxicilina PREOP es efectiva en prevenir fracasos implantológicos tempranos, pero no reduce significativamente el riesgo de infección, así como la prescripción POSOP

La prescripción PREOP única y PREOP + POSOP presentan tasas de fracaso implantológico e infecciones POSOP similares, reduciendo ambas de manera significativa el riesgo de fracaso temprano en condiciones normales

La profilaxis ATB reduce un $2 \%$ la tasa de fracaso implantológico temprano, sin embargo, en condiciones normales, no muestra un beneficio añadido frente a no realizarla

No demostrables los beneficios de la irrigación con solución antibiótica o la administración sistémica de antibióticos en alvéolos infectados

La profilaxis ATB, en condiciones normales, no muestra un beneficio añadido frente a no realizarla La profilaxis ATB reduce significativamente la tasa de fracaso temprano de implantes, pero no el riesgo de infección

La profilaxis ATB, en concreto, 2-3 g de amoxicilina PREOP, disminuye el fracaso temprano de implantes dentales, pero no el riesgo de infección o eventos adversos

Las tasas de fracaso temprano de implantes inmediatos anuales son menores al administrar antibióticos profilácticos POSOP durante 5-7 días $(0,51 \%)$ en comparación a la prescripción PREOP única $(1,87 \%)$ o PERIOP $(0,75 \%)$

Los autores se muestran a favor de la prescripción sistémica de antibióticos PERIOP en estos casos La prescripción de $2 \mathrm{~g}$ de amoxicilina PREOP reduce significativamente las tasas de fracaso de implantes dentales en condiciones normales. Sin embargo, no sientan la prescripción de realizar profilaxis ATB de manera rutinaria en implantología oral ni cual es el ATB de elección

PREOP: preoperatoria. POSOP: posoperatoria. PERIOP: perioperatoria. ECA: ensayos clínicos aleatorizados. EC: ensayos clínicos. ATB: antibiótico.g: gramo [s].
En general, los estudios que analizan las diferentes revisiones sistemáticas mostraron posologías de administración muy heterogéneas. Salvo unos pocos ensayos clínicos que analizaron otros antibióticos, como cefalosporinas de primera generación ${ }^{2}$, feniticilina ${ }^{27}$ o fenoximetilpenicilina (1 gramo preoperatorio seguido de 1 gramo, 3 veces al día, durante 10 días $)^{8}$, el antibiótico más estudiado fue la amoxicilina con diversas posologías: $2^{28-33}$ o 3 gramos preoperatorios de amoxicilina, 1 gramo preoperatorio y $500 \mathrm{mg}$ posoperatorios 4 veces al día durante dos días ${ }^{24,34}, 2$ gramos preoperatorios seguidos de 2 gramos dos veces al día durante 7 días $^{30}, 1$ gramo preoperatorio y dos gramos al día durante dos días ${ }^{35}, 2$ gramos posoperatorios administrados de manera única ${ }^{32}, 2$ gramos de amoxicilina comenzando tras la cirugía durante 7 días ${ }^{30}$, 2 gramos preoperatorios seguidos de $500 \mathrm{mg}$ dos veces al día durante 3 días $^{32}$ y 1 gramo de amoxicilina posoperatorio, dos veces al día durante 7 días.

\section{DISCUSIÓN}

\section{Evidencia disponible}

En los últimos años se han publicado una gran multitud de estudios acerca de los efectos de la profilaxis antibiótica en el fracaso temprano (es decir, al fracaso del implante de manera previa a la carga protésica del mismo, lo que indica el fallo del proceso de osteointegración), en la prevención de infecciones y/o en la disminución de la morbilidad tras tratamientos implantológicos; sin embargo, la heterogeneidad 
entre los diferentes estudios hace difícil su comparación. En concreto, diversos estudios han analizado los resultados obtenidos tras: a) una profilaxis antibiótica única, de manera preoperatoria comparado con un placebo; b) tras una profilaxis perioperatoria, es decir, de manera previa, continuando varios días tras la inserción del implante; y c) tras una profilaxis posoperatoria. Además, la realización de procedimientos de regeneración ósea ${ }^{31}$, la inclusión de pacientes fumadores $28,29,31,32,35$, la humectación de los implantes con plasma rico en factores de crecimiento (PRFG) ${ }^{31}$, la inserción de implantes con diferentes diseños y tratamientos de superficie, así como la inserción de implantes inmediatos ${ }^{28,29}$ pueden actuar como factores de confusión.

\section{Fracaso temprano de implantes}

Diversas revisiones sistemáticas ${ }^{5,21}$ y metanálisis ${ }^{4,23,24}$ muestran que la administración de una única dosis de 2 gramos de amoxicilina una hora antes de la cirugía, en pacientes sanos, disminuye significativamente la incidencia de fracaso temprano de los implantes. Sin embargo, en su metanálisis, Chrcanovic y cols. ${ }^{4}$ observaron que no existían diferencias estadísticamente significativas al descartar aquellos estudios considerados de alto riesgo de sesgo.

En cuanto a la posología, en general no se han visto diferencias entre la prescripción de una única dosis de 2 gramos de amoxicilina, una hora antes de la intervención, comparado con continuar dicha pauta con $500 \mathrm{mg}$, 4 veces al día durante dos días ${ }^{7,24}$. Por este motivo, no se ha observado un beneficio mayor al prolongar el tratamiento antibiótico más allá del día de la cirugía.

\section{Morbilidad}

La profilaxis antibiótica en cualquiera de sus posologías no disminuye el riesgo de infección $n^{4,21,23,24}$, ni tampoco se observaron diferencias estadísticamente significativas, en cuanto a reacciones adversas entre grupos placebo y control ${ }^{7,24}$. Estas reacciones adversas secundarias al tratamiento con antibióticos fueron leves en la mayoría de los casos, como somnolencia, diarrea ${ }^{7,34}$, hinchazón abdominal, acidez estomacal y rash cutáneo, aunque también se han descrito casos de reacciones severas como edema laríngeo ${ }^{33}$ y reacciones de hipersensibilidad ${ }^{34}$.

Por otro lado, Singh-Gill y cols..$^{19}$ determinaron un riesgo relativo de complicaciones posoperatorias de 0,74 inherente a la profilaxis antibiótica en implantología oral, es decir, que el uso preventivo de antibióticos probablemente reduce el riesgo de complicaciones tras la cirugía implantológica en un $26 \%$.

\section{Indicaciones de la profilaxis antibiótica en implantología oral}

Varios metanálisis muestran que cada $25-50$ pacientes tratados con profilaxis antibiótica, solamente en uno de ellos se evitará un fracaso temprano $4,5,23,24$. Según Lund y cols. ${ }^{5}$, esta reducción del riesgo es del $2 \%$. Sin embargo, el costo y los riesgos del uso de antibióticos siempre deben sopesarse frente a la gravedad de la afección que se debe prevenir. Por lo tanto, la afirmación de que la profilaxis antibiótica preoperatoria reduce significativamente las pérdidas de implantes no necesariamente lleva a su adopción como un protocolo estándar en implantología oral. El valor de esta reducción del riesgo de fracaso temprano debe situarse en el contexto de los problemas emergentes con la resistencia a antibióticos antes de poder formular directrices sólidas. La prevalencia de infecciones posoperatorias es del 5,9 $\%{ }^{24}$, pudiendo afectar hasta al 2,4 \% de los implantes y, de estos, dos tercios fracasarán (la mayoría antes de la carga protésica) ${ }^{36}$. A la luz de estos resultados se debe valorar el coste biológico del fracaso de los implantes por encima del coste económico producido, ya que el miedo a la infección del lecho quirúrgico y a las repercusiones legales y económicas motivan en la gran mayoría de casos la realización de profilaxis antibiótica ${ }^{37}$. En general, aquellos casos en los que estaría indicada la realización de una profilaxis antibiótica en tratamientos de implantes dentales serían: a) en pacientes en riesgo de sufrir una endocarditis bacteriana $a^{1,7} ; b$ ) en pacientes inmunodeprimidos o con determinadas alteraciones sistémicas $^{3-5,7}$; c) en implantes inmediatos; d) lechos infectados; e) en cirugías extensas y prolongadas, y f) en cirugías regenerativas por un mayor riesgo de dehiscencia mucosa, debido a un mayor riesgo de infección asociada en estos casos ${ }^{7}$.

\section{Inserción de implantes en pacientes sanos sin condicionantes añadidos}

Según diversas revisiones sistemáticas, no existe un beneficio añadido en la prescripción profiláctica de antibióticos en pacientes sanos, con casos de implantes favorables y una adecuada calidad ósea del lecho quirúrgico ${ }^{4,5,7,19}$. Únicamente el estudio de Chen y cols. ${ }^{21}$ mostró resultados positivos en estos pacientes en cuanto a la reducción del fracaso del tratamiento implantológico e infecciones posoperatorias, lo cual está en discordancia con el resto de literatura disponible. En general, no existe una evidencia significativa que apoye la profilaxis antibiótica de manera rutinaria con el fin de prevenir el fracaso temprano y las infecciones posoperatorias de implantes dentales ${ }^{19}$

\section{Inserción de implantes en pacientes sanos con patologías médicas concomitantes}

En este grupo se incluiría a pacientes con factores de riesgo de infección local o sistémica: son pacientes oncológicos, inmunosuprimidos, trastornos metabólicos y pacientes con insuficiencia renal o hepática ${ }^{38}$.

\section{Inserción de implantes en lechos infectados}

La evidencia muestra que los implantes dentales pueden ser insertados en alvéolos posextracción con infecciones periapicales o periodontales con tasas de éxito similares a aquellos insertados en alvéolos no infectados o en hueso pristino. Waasdorp y cols. ${ }^{26}$ llevaron a cabo una revisión sistemática con el fin de establecer una serie de recomendaciones acerca de 
la inserción inmediata de implantes en estos casos y, a pesar de que todos los estudios analizados emplearon antibióticos sistémicos perioperatorios, los autores se mostraron a favor de su prescripción, al menos hasta que nuevos ensayos clínicos demuestren lo contrario. Otros autores no han podido probar los beneficios de la irrigación con solución antibiótica o la administración sistémica de antibióticos en alvéolos infectados ${ }^{22}$.

\section{Inserción inmediata de implantes}

Lang y cols. ${ }^{25}$ analizaron las tasas de supervivencia y de fracaso de implantes inmediatos con seguimientos de, al menos, un año, mostrando valores medios de 98,4 y 0,82 \%, respectivamente. Tras tener en cuenta diversos factores (motivo de las exodoncias, prescripción de antibióticos, posición de los implantes [anterior o posterior, mandibular o maxilar] y tipo de carga) únicamente el régimen antibiótico influyó de manera significativa en las tasas de supervivencia. Los diversos autores emplearon diversas posologías: una única dosis preoperatoria, 5-7 días posoperatorios, y una dosis única preoperatoria seguido de 5-7 días posoperatorios. Los resultados mostraron tasas anuales de fracaso del 1,87 \%, 0,51 \% y 0,7 5\%, respectivamente. Por tanto, en casos de inserción inmediata de implantes dentales se recomienda la prescripción posoperatoria de antibióticos durante 5-7 días.

\section{Elevaciones de seno maxilar}

La incidencia de infecciones posoperatorias tras elevaciones sinusales es baja (2-5,6 \%) sin que se establezca la diferencia entre infecciones por sinusitis o sinusitis verdaderas. Es decir, en estos casos, si existe una integridad de la membrana y acontece una infección, esta tendrá su origen en el injerto subantral. A pesar de ser infrecuentes, este tipo de infecciones ponen en riesgo la regeneración realizada y la supervivencia de los implantes ${ }^{39}$. La extensión de la cirugía, la respuesta del huésped y la posible contaminación quirúrgica esperada, así como las consecuencias de una infección en los senos paranasales, condicionan la necesidad de profilaxis antibiótica ${ }^{14}$, sobre todo en casos de elevaciones sinusales mediante el abordaje con ventana lateral. Por este motivo, si ocurre una complicación, es importante la biodisponibilidad de antibiótico en sangre durante el momento de la contaminación, y esto se logra mediante una profilaxis antibiótica preoperatoria que permita lograr el pico de concentración sérica del antibiótico durante la intervención quirúrgica ${ }^{40}$.

\section{Cirugías óseas regenerativas}

Algunos autores recomiendan la prescripción de antibióticos en procedimientos de regeneración ósea de manera previa y posterior a la cirugía con el fin de asegurar concentraciones séricas adecuadas hasta que el injerto disponga de su propia vascularización, es decir, comenzando una hora antes de la intervención y continuando durante los 9 días posteriores ${ }^{14}$. Maurece y cols. ${ }^{41}$, tras analizar partículas óseas autólogas extraídas de pacientes en los que se había hecho, o no, una profilaxis previa, observaron que todas presentaban contaminación bacteriana. Los grupos más habituales fueron Streptococcus, más concretamente S. constellatus y S. mitis, considerados como potenciales responsables de infecciones odontógenas. Estos autores también observaron que la realización de una profilaxis previa reduce, pero no elimina, la contaminación bacteriana de las partículas óseas recolectadas durante la preparación del lecho quirúrgico. Pese a ello, la tasa de infección posterior a estos procedimientos es baja ( $4 \%$ ), con o sin la administración de una profilaxis antibiótica. La evidencia al respecto, basada en dos ensayos clínicos prospectivos controlados a doble ciego con un grupo placebo, comparado con la profilaxis antibiótica con una única dosis de $2 \mathrm{~g}$ de feneticilina ${ }^{27}$ o de dos gramos preoperatorios de una cefalosporina de primera generación seguido de 1 gramo al día, 3 veces al día, durante 3 días², mostraron que no existen diferencias significativas entre ambos grupos en cuanto a las tasas de infección posoperatorias. En concreto, el estudio de Lindeboom y van den Akker ${ }^{27}$ arrojó unos resultados significativos a favor de la profilaxis antibiótica; sin embargo, Brenann y cols. ${ }^{42}$, en una carta al Editor, refutaron dichos resultados debido a una incorrecta selección del método estadístico que, tras corregirlo, no mostraba una significancia estadística. En general, se recomienda la prescripción de aminopenicilinas e inhibidores de la betalactamasa ${ }^{43}$.

\section{Prevención frente a la endocarditis bacteriana}

A pesar de que la boca constituye un campo quirúrgico séptico, Piñeiro y cols. ${ }^{44}$ observaron que la inserción de implantes, con y sin elevación de colgajos mucoperiósticos, no representa un riesgo significativo de bacteriemia, por lo que arroja dudas sobre la necesidad de profilaxis antibiótica en pacientes considerados en riesgo de desarrollar una endocarditis bacteriana $\mathrm{u}$ otras infecciones focales. A pesar de ello, actualmente la profilaxis antibiótica estaría indicada en estos casos ya que, de producirse, puede constituir un compromiso vital para el paciente ${ }^{45}$.

Se ha estimado que la frecuencia de bacteriemia transitoria tras un tratamiento dental varía en función del procedimiento: extracción dental (10-100 \%), cirugía periodontal (36-88 \%), raspado y alisado radicular (8-80 \%) o profilaxis (mayor al $40 \%$ ). Sin embargo, son equivalentes a procedimientos rutinarios realizados por el propio paciente, como el cepillado y el uso de seda dental (20-68\%), irrigadores dentales (7-50\%) y la masticación de alimentos pegajosos (7-51\%). Teniendo en cuenta que los procedimientos dentales provocan una magnitud de bacteriemia relativamente baja $\left(<10^{4} \mathrm{UFC} / \mathrm{mm}\right)$ y similares a actividades rutinarias realizadas varias veces al día por pacientes periodontalmente sanos, el riesgo de padecer una endocarditis bacteriana por un procedimiento dental, como la cirugía de implantes, es baja ${ }^{45}$. Por otro lado, estudios recientes han mostrado que la profilaxis con amoxicilina reduce de manera significativa la naturaleza, incidencia y duración de la bacteriemia ${ }^{46,47}$, sin embargo no existe evidencia que indique que dicha reducción disminuya el riesgo o prevenga la endocarditis bacteriana ${ }^{45}$. 
El periodo de tiempo en el que aparecen los síntomas de endocarditis (fiebre y otros signos de infección sistémica) tras una bacteriemia, es de 7 (78\% de los casos) a 14 días (85 \%) para Streptococcus del grupo viridans o Enterococcus ${ }^{48}$, aunque actualmente ha habido un repunte de los casos originados por Staphylococcus ${ }^{49,50}$, por lo que su relación con tratamientos dentales se ha sobreestimado ${ }^{48}$. Más aún, solo un número extremadamente pequeño de casos de endocarditis bacteriana podría prevenirse con profilaxis antibiótica, incluso si fuera $100 \%$ efectiva. Por tanto, parece más importante lograr, previo al tratamiento con implantes, un correcto control de placa bacteriana y tratar infecciones preexistentes con el fin de reducir la bacteriemia secundaria a los procedimientos diariamente realizados por el paciente, priorizando el establecimiento de condiciones de higiene bucal adecuadas, frente a la celeridad de los tratamientos y las exigencias de los pacientes frente a los mismos. No se ha podido demostrar que la profilaxis antibiótica prevenga la posible endocarditis derivada de una bacteriemia inducida por un procedimiento dental, ya que los datos derivan de ensayos únicos aleatorizados o estudios no aleatorizados (nivel de evidencia B) ${ }^{45}$, pero si así fuera, sería razonable que fuera limitado únicamente a aquellos procedimientos dentales que supongan la manipulación o perforación gingival o mucosa (incluida la retirada de puntos de sutura), como el tratamiento con implantes en pacientes que tengan planificada una intervención cardiaca valvular o aquellos con determinadas patologías cardiacas, ya que exhiben el mayor riesgo de endocarditis ${ }^{51}$ :

- Portadores de una válvula cardiaca o material protésico usado para la reparación de válvulas cardiacas.

- Antecedentes de endocarditis bacteriana.

- Enfermedad cardiaca congénita (ECC): ECC cianótica no tratada; ECC tratada mediante material protésico durante los seis meses siguientes a la intervención; y en el caso anterior en el que persistan defectos residuales del área a tratar. - Valvulopatías desarrolladas tras trasplante cardiaco.

Por lo tanto, la prescripción de antibióticos profilácticos, fundamentalmente estaría justificada desde el punto de vista legal ${ }^{13}$. Más aún, el tratamiento de una endocarditis requiere la prescripción de antibióticos intravenosos de manera prolongada durante su fase activa y, aproximadamente, la mitad de los pacientes requerirán una cirugía valvular responsable de una alta mortalidad ( $40 \%$ a los 4 años), por lo que parece razonable la prescripción profiláctica de antibióticos ${ }^{49,50}$. Según la American Heart Association ${ }^{45}$, en este grupo de pacientes se recomienda una única dosis, 30 a 60 minutos antes de la intervención. Si se olvidó realizarla, se podría realizar hasta 2 h después de la intervención. Si el paciente ya está en tratamiento a largo plazo con antibióticos, se recomienda prescribir otro de diferente tipo de acción con el fin de potenciar al antibiótico actual.

\section{Selección del antibiótico y mecanismo de acción}

El mecanismo por el cual la profilaxis antibiótica puede influir positivamente en las tasas de fracaso temprano de los implantes aún es desconocido. La realización de una incisión mucosa y la inserción de un implante dental rompe la barrera protectora mucosa, permitiendo la invasión y contaminación bacteriana, lo que puede conducir a la infección de la herida y a un mayor riesgo de infección. La American College of Surgeons (Committee on Control of Surgical Wound Infections) desarrolló una clasificación de las heridas quirúrgicas y el riesgo de infección. En esta clasificación, los procedimientos óseos e implantológicos se englobarían dentro de la clase 2 ("herida limpia-contaminada"), las cuales muestran unas tasas de infección asociadas del 10 al $15 \%$. En este sentido, los antibióticos profilácticos en combinación con antisépticos orales podrían crear un medio ambiente local más aséptico durante la inserción de implantes y en el posoperatorio inmediato que pueden mejorar la cicatrización y, de manera ulterior, la osteointegración ${ }^{52}$.

Los diferentes estudios han fallado en relacionar la prescripción profiláctica de antibióticos con una reducción del dolor posoperatorio, de la inflamación y una mejoría en la cicatrización del lecho quirúrgico. Asimismo, tampoco se ha podido relacionar con una reducción del riesgo de infección, lo que puede ser explicado porque, en algunas ocasiones, se producen infecciones asintomáticas, sin aparentes signos clínicos asociados que pueden determinar la pérdida de algunos implantes ${ }^{53,54}$. Con todo, las tasas de infección son bajas incluso sin la prescripción de antibióticos. Este mismo grupo de trabajo puso de manifiesto los diferentes tipos de antibióticos y posologías prescritas por los odontólogos del Reino Unido, sin embargo, una de las conclusiones extraídas es que la duración de la profilaxis antibiótica debe ser lo más corta posible para que sea efectiva. En este sentido, una sola dosis preoperatoria de antibiótico es suficiente. En casos en los que la intervención se extienda en el tiempo o el daño sea considerable se podrían administrar otras dosis de antibiótico ${ }^{38,55}$.

La amoxicilina es el antibiótico más comúnmente recetado por dentistas debido a su gran absorción ${ }^{51}$ y a su efectividad contra la mayor parte de los patógenos orales ${ }^{56}$, seguido de la clindamicina en pacientes alérgicos a penicilinas ${ }^{51}$ (aproximadamente el $10 \%$ de la población presenta alergia a penicilinas ${ }^{57}$ ). Sin embargo, la evidencia disponible no se ha decantado sobre qué antibiótico destinado a estos fines es el más efectivo ${ }^{19}$. En general, los diferentes estudios analizan la efectividad de diversas posologías de amoxicilina y, únicamente en pacientes alérgicos, emplean clindamicina, pero no existe evidencia que analice específicamente el uso de clindamicina como antibiótico profiláctico en implantología oral.

La amoxicilina, tras ser administrada vía oral, alcanza unos niveles plasmáticos máximos en 1-2 horas y su semivida de eliminación (o tiempo que tarda en eliminarse el 50 \% de su concentración plasmática) es de 1-1,5 horas ${ }^{58}$, por lo que varias horas más tarde, su presencia en el fluido crevicular gingival está por debajo de los límites detectables ${ }^{59,60}$ y a las 24 horas no es detectable a nivel sérico ${ }^{61}$. Por tanto, la amoxicilina es efectiva en reducir la flora oral hasta 12 horas posteriores a su administración ${ }^{41}$. Un reciente estudio llevado a cabo por Aravena y cols. $^{62}$ (2018) ha concluido que la administración de 1 gramo de amoxicilina una hora antes de la cirugía implantológica es suficiente para alcanzar la concentración mínima inhibitoria (CMI) de 5 mg/l recomendada por la National Committee for Clinical Laboratory Standards (NCCLS) para bacterias anaerobias ${ }^{63}$. En concreto, a la 1,16 horas ( \pm 0,37 horas) de la administración 
de un gramo de amoxicilina, su concentración en la sangre venosa es de $421 \pm 212 \mathrm{mg} / \mathrm{l}$ y, de $500 \pm 263 \mathrm{mg} / \mathrm{l}$ en el lecho del implante ${ }^{62}$. La efectividad del antibiótico se alcanza cuando se supera la CMI para una bacteria determinada de dos a cuatro veces $^{64}$. Exceder dicho rango terapéutico crea una ventana de sobredosis terapéutica conocida como "ventana de selección de mutantes" ${ }^{65}$, que modifica la susceptibilidad de las bacterias a los antibióticos, haciéndolas resistentes ${ }^{62}$.

La clindamicina alcanza su concentración sérica máxima a los 45-60 minutos de haberse administrado en adultos. En sujetos con una función renal normal, su semivida de eliminación es de 2-3 horas. Por ello, el antibiótico profiláctico tiene como objeto la prevención de la contaminación y diseminación de una infección quirúrgica ${ }^{66}$.

Por otro lado, la azitromicina, tras una profilaxis antibiótica preoperatoria única de $500 \mathrm{mg}$, se encuentra en concentraciones de 224 y 203 mg/l en fluido crevicular gingival y periimplantario, respectivamente, a los 13 días posquirúrgicos, por lo que puede ser interesante su uso como antibiótico profiláctico, ya que tiene importantes efectos sobre la inflamación y la cicatrización temprana tras la inserción de implantes, disminuyendo los niveles del factor estimulante de colonias de granulocitos (G-CSF), interleucinas 6 y 8, proteína inflamatoria de macrófa-

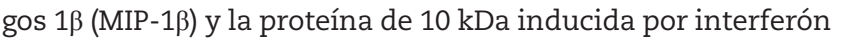
(IP-10), reduciendo la movilización de precursores de granulocitos y el reclutamiento de células inmunes e inflamatorias durante la fase de curación y presenta una biodisponibilidad mayor que la amoxicilina y la clindamicina ${ }^{60}$. A pesar de que los neutrófilos juegan un papel fundamental en la curación, la reducción de una excesiva infiltración se asocia a una cicatrización acelerada del lecho quirúrgico en ratones ${ }^{67}$. Durante el proceso de cicatrización, los tejidos blandos perimplantarios se adhieren al implante y a los dientes adyacentes, lo que dificulta la penetración bacteriana y ayuda en la resolución de la infección. Clínicamente se manifiesta con una reducción en el volumen del fluido crevicular gingival y mucoso.

Otros antibióticos fueron estudiados, como la fenoximetilpenicilina (1 gramo preoperatorio seguido de 1 gramo, 3 veces al día durante 10 días) ${ }^{8}$, la feneticilina (2 g preoperatorios $)^{27}$, o una cefalosporina de $1 .^{a}$ generación (dos gramos preoperatorios seguido de 1 gramo al día, 3 veces al día, durante 3 días) ${ }^{2}$, no observando diferencias significativas entre dichos grupos y los grupos placebo en cuanto a la tasa de infección posoperatoria.

\section{Prevención de infecciones}

Existen diversos factores que pueden contribuir a un riesgo de infección posoperatoria y fracaso temprano aumentados. Estos factores dependen del estado médico del paciente que, en algunos casos, puede conllevar alteraciones en la cicatrización de heridas, el hábito tabáquico, un inadecuado grado de asepsia y una técnica quirúrgica inadecuada, y la contaminación bacteriana durante la inserción de los implantes ${ }^{68}$, pero los factores etiológicos más importantes en el fracaso temprano son el trauma quirúrgico en combinación con condiciones anatómicas desfavorables.

El mantenimiento de un campo quirúrgico lo más aséptico posible es uno de los factores quirúrgicos clave en el trata- miento con implantes dentales. Uno de los antisépticos más recomendados es el digluconato de clorhexidina, ya que se ha visto que enjuagándose con una concentración al 0,12 \% de manera prequirúrgica reduce la tasa de pérdida implantaria del $13,5 \%$ al $4,4 \%$ en pacientes con diabetes tipo $2{ }^{69} \mathrm{y}$, además, reduce de manera significativa el crecimiento del biofilm sobre los pilares de cicatrización, en comparación a un grupo placebo ${ }^{70}$.

Para evitar un uso indebido de los antibióticos y la necesidad de una antibioterapia terapéutica por infecciones posoperatorias, el implantólogo debería: 1) informar a los pacientes sobre cómo tomar los antibióticos correctamente según la posología prescrita, y sensibilizarlos en los peligros de su uso indebido; 2) proporcionarles información acerca de cómo prevenir las infecciones posoperatorias; 3) reducir al mínimo la contaminación quirúrgica del lecho intervenido, velando por la limpieza del entorno y la esterilidad del instrumental y material empleados; 4) prescribir antibióticos únicamente cuando estén indicados, y 5) notificar las infecciones resistentes a los antibióticos a los organismos de farmacovigilancia 6 .

Otro de los factores a tener en cuenta es que la profilaxis antibiótica en muchos casos no es efectiva, debido a que los pacientes pueden presentar resistencias microbianas. En este sentido, Classen y cols. ${ }^{40}$ evaluaron la efectividad de diferentes protocolos de profilaxis antibiótica observando que, en los 43 pacientes a los que se les aislaron muestras microbiológicas, 25 (o el 58 \%) eran resistentes a los antibióticos empleados.

Por este motivo es fundamental controlar los aspectos que rodean al tratamiento con implantes, llevando a cabo medidas preventivas frente a infecciones:

- Cese del hábito tabáquico en pacientes fumadores.

- Resolución de patologías bucales previas.

- Adecuado control sistémico del paciente ${ }^{39}$ : diabetes mellitus, estados de inmunosupresión, terapia con corticoesteroides de larga duración, malnutrición, obesidad y población de edad avanzada, son factores que se han relacionado con un mayor riesgo de infección ${ }^{71}$.

- Control del estado periodontal del paciente: se debe de resolver cualquier patología periodontal previa y se recomienda que los niveles de placa bacteriana y de índice de sangrado al sondaje se encuentren por debajo del $15 \%$, para lo cual son recomendables los enjuagues con antisépticos, como el digluconato de clorhexidina, de manera previa a la cirugía y durante el periodo de cicatrización.

- Asepsia del campo quirúrgico y desinfección de la piel con una solución antiséptica (como povidona yodada).

- Prevenir el sobrecalentamiento del lecho quirúrgico.

- En el caso de realizar regeneraciones óseas: prevenir la contaminación por saliva del injerto óseo y/o de otros materiales $^{39}$, ya que la carga bacteriana en la misma es muy elevada $\left(10^{9} \mathrm{CFU} / \mathrm{ml}\right)^{41}$ y emplear dos sets de instrumentos: uno para para elevación del colgajo y otro para la fase regenerativa.

- Limpiar el campo quirúrgico con solución salina estéril, y lograr una adecuada hemostasia intra y posquirúrgica.

- Reducir los tiempos quirúrgicos lo máximo posible ${ }^{39}$ : las intervenciones que duran menos de 1 hora tienen una incidencia de infección de un 1,3\%, mientras que aquellas que duran 3 horas del $4 \%^{71}$.

- Adecuado tratamiento farmacológico posoperatorio. 
- Correcto protocolo de revisiones posquirúrgicas: semanalmente durante el primer mes y mensualmente durante los siguientes 3 meses $^{39}$.

Las futuras vías de investigación se deberían de centrar en el efecto de los antibióticos sobre la respuesta inflamatoria en las primeras fases de la cicatrización y cómo pueden influir sobre la osteointegración, así como el antibiótico de elección en implantología oral y su posología ya que, debido a la falta de evidencia al respecto, la tendencia de los implantólogos es a prescribir antibióticos basándose en su experiencia. También sería interesante conocer el impacto de la profilaxis antibiótica en la prevención del fracaso temprano de implantes dentales y, en el riesgo de infección posoperatoria en pacientes médicamente comprometidos, en los que el riesgo de infección es mayor. Asimismo, son necesarios más estudios que analicen diversos fármacos antibióticos para los mismos fines, con el objetivo de determinar cuál es el más efectivo en tratamientos con implantes dentales.

\section{CONCLUSIONES}

Aunque existe evidencia de que la prescripción de 2 g de amoxicilina (o en alérgicos, $600 \mathrm{mg}$ de clindamicina) una hora antes de la cirugía de implantes reduce de manera significativa el riesgo de fracaso temprano, se debería de llevar a cabo una prescripción responsable de los antibióticos.

La utilización profiláctica de antibióticos deberá ser restringida a aquellos casos en los que las consecuencias de una infección de origen bacteriano intraoral sean potencialmente graves. Estos casos son pacientes en riesgo de sufrir una endocarditis bacteriana, pacientes inmunodeprimidos, en la inserción de implantes inmediatos y en lechos infectados, en cirugías extensas y prolongadas, y en cirugías regenerativas por un mayor riesgo de dehiscencia mucosa. Su prescripción en pacientes sanos, sin condicionantes anatómicos y en lechos quirúrgicos con una correcta calidad ósea no estaría justificado.

\section{FUENTE DE FINANCIACIÓN}

La presente investigación no ha recibido ninguna beca específica de agencias de los sectores público, comercial o sin ánimo de lucro.

\section{CONFLICTO DE INTERESES}

Los autores declaran no tener ningún conflicto de intereses.

\section{B I B L I O G R A F Í A}

1. Canadian Dental Association. CDA Position on the Prevention of Infective Endocarditis; 2014.

2. Lee JW, Lee JY, Kim SM, Kim MJ, Lee JH. Prophylactic antibiotics in intra-oral bone grafting procedures: a prospective, randomized, double-blind clinical trial. J Korean Assoc Oral Maxillofac Surg. 2012;38(2):90-5. DOI: 10.5125/jkaoms.2012.38.2.90.
3. Ahmad N, Saad N. Effects of antibiotics on dental implants: a review. J Clin Med Res. 2012;4(1):1-6. DOI: 10.4021/jocmr658w.

4. Chrcanovic BR, Albrektsson T, Wennerberg A. Prophylactic antibiotic regimen and dental implant failure: a meta-analysis. J Oral Rehabil. 2014;41(12):941-56. DOI: 10.1111/joor.12211.

5. Lund B, Hultin M, Tranaeus S, Naimi-Akbar A, Klinge B. Complex systematic review - Perioperative antibiotics in conjunction with dental implant placement. Clin Oral Implants Res. 2015;26(Suppl. 11):1-14. DOI: 10.1111/clr.12637.

6. Organización Mundial de la Salud. Resistencia a los antibióticos; 2017.

7. Esposito M, Worthington HV, Loli V, Coulthard P, Grusovin MG. Interventions for replacing missing teeth: antibiotics at dental implant placement to prevent complications. Cochrane Database Syst Rev. 2010:CD004152. DOI: 10.1002/14651858.CD004152.pub3.

8. Gynther GW, Kondell PA, Moberg LE, Heimdahl A. Dental implant installation without antibiotic prophylaxis. Oral Surg Oral Med Oral Pathol Oral Radiol Endod. 1998;85(5):509-11.

9. Ziment I. Complications of antibiotic therapy. Calif Med. 1972;117(5):24-48.

10. ECDC/EMEA Joint Technical Report. The Bacterial Challenge: Time to React; 2009.

11. Matesanz P, Figuero E, Gimenez MJ, Aguilar L, Llor C, Prieto J, et al. [From knowledge of bacterial etiology to the treatment and prevention of the most prevalent infections in the community: odontological infections]. Rev Esp Quimioter. 2005;18(2):136-45.

12. Gutiérrez J, Bagán J, Bascones A, Llamas R, Llena J, Morales A, et al. Documento de consenso sobre la utilización de profilaxis antibiótica en cirugía y procedimientos dentales. Rev Esp Cir Oral Maxilofac. 2006;28:151-81.

13. Ireland RS, Palmer NO, Lindenmeyer A, Mills N. An investigation of antibiotic prophylaxis in implant practice in the UK. Br Dent J. 2012;213(8):E14. DOI: 10.1038/sj.bdj.2012.960.

14. Froum SJ, Weinberg MA. An Evaluation of Antibiotic Use in Periodontal and Implant Practices. Int J Periodontics Restorative Dent. 2015;35(4):481-7. DOI: 10.11607/prd.2488.

15. Pyysalo M, Helminen M, Antalainen A-K, Sandor GK, Wolff J. Antibiotic prophylaxis patterns of Finnish dentists performing dental implant surgery. Acta Odontol Scand. 2014;72(8):806-10. DOI: 10.3109/00016357.2014.913194.

16. Datta R, Grewal Y, Bath K, Singh A. Current Trend of Antimicrobial Prescription for Oral Implant Surgery Among Dentists in India. J Maxillofac Oral Surg. 2014;13(4):503-7. DOI: 10.1007/ s12663-013-0567-7.

17. Khalil D, Hultin M, Andersson Fred L, Parkbring Olsson N, Lund B. Antibiotic prescription patterns among Swedish dentists working with dental implant surgery: adherence to recommendations. Clin Oral Implants Res. 2015;26(9):1064-9. DOI: 10.1111/clr.12402.

18. Deeb GR, Soung GY, Best AM, Laskin DM. Antibiotic Prescribing Habits of Oral and Maxillofacial Surgeons in Conjunction With Routine Dental Implant Placement. J Oral Maxillofac Surg. 2015;73(10):1926-31. DOI: 10.1016/j.joms.2015.05.024.

19. Singh Gill A, Morrissey H, Rahman A. A Systematic Review and Meta-Analysis Evaluating Antibiotic Prophylaxis in Dental Implants and Extraction Procedures. Medicina (Kaunas). 2018;54(6). pii: E95. DOI: 10.3390/medicina54060095.

20. Rodríguez Sánchez F, Rodríguez Andres C, Arteagoitia I. Which antibiotic regimen prevents implant failure or infection after dental implant surgery? A systematic review and meta-analysis. J Craniomaxillofac Surg. 2018;46(4):722-36. DOI: 10.1016/j. jcms.2018.02.004

21. Chen Z, Chen D, Zhang S, Tang L, Li Q. Antibiotic prophylaxis for preventing dental implant failure and postoperative infection: A systematic review of randomized controlled trials. Am J Dent. 2017;30:89-95.

22. Chrcanovic BR, Martins MD, Wennerberg A. Immediate placement of implants into infected sites: a systematic review. Clin Implant Dent Relat Res. 2015;17:e1-16. DOI: 10.1111/cid.12098. 
23. Ata-Ali J, Ata-Ali F, Ata-Ali F. Do antibiotics decrease implant failure and postoperative infections? A systematic review and meta-analysis. Int J Oral Maxillofac Surg. 2014;43(1):68-74. DOI: 10.1016/j.ijom.2013.05.019.

24. Esposito M, Grusovin MG, Worthington HV. Interventions for replacing missing teeth: antibiotics at dental implant placement to prevent complications. Cochrane Database Syst Rev. 2013;(7):CD004152. DOI: 10.1002/14651858.CD004152.pub4.

25. Lang NP, Pun L, Lau KY, Li KY, Wong MCM. A systematic review on survival and success rates of implants placed immediately into fresh extraction sockets after at least 1 year. Clin Oral Implants Res. 2012;23(Suppl. 5):39-66. DOI: 10.1111/j.1600-0501.2011.02372.x.

26. Waasdorp JA, Evian CI, Mandracchia M. Immediate placement of implants into infected sites: a systematic review of the literature. J Periodontol. 2010;81(6):801-8. DOI: 10.1902/jop.2010.090706.

27. Lindeboom JAH, van den Akker HP. A prospective placebocontrolled double-blind trial of antibiotic prophylaxis in intraoral bone grafting procedures: a pilot study. Oral Surg Oral Med Oral Pathol Oral Radiol Endod. 2003;96(6):669-72. DOI: 10.1016/S1079210403005559.

28. Esposito M, Cannizzaro G, Bozzoli P, Consolo U, Felice P, Ferri V, et al. Efficacy of prophylactic antibiotics for dental implants: a multicentre placebo-controlled randomised clinical trial. Eur J Oral Implantol. 2008;1(1):23-31.

29. Esposito M, Cannizzaro G, Bozzoli P, Checchi L, Ferri V, Landriani S, et al. Effectiveness of prophylactic antibiotics at placement of dental implants: a pragmatic multicentre placebocontrolled randomised clinical trial. Eur J Oral Implantol. 2010;3(2):135-43.

30. Caiazzo A, Casavecchia P, Barone A, Brugnami F. A pilot study to determine the effectiveness of different amoxicillin regimens in implant surgery. J Oral Implantol. 2011;37(6):691-6. DOI: 10.1563/AAID-JOI-D-09-00134.1.

31. Anitua E, Aguirre JJ, Gorosabel A, Barrio P, Errazquin JM, Roman $\mathrm{P}$, et al. A multicentre placebo-controlled randomised clinical trial of antibiotic prophylaxis for placement of single dental implants. Eur J Oral Implantol. 2009;2(4):283-92.

32. Tan WC, Ong M, Han J, Mattheos N, Pjetursson BE, Tsai AYM, et al. Effect of systemic antibiotics on clinical and patientreported outcomes of implant therapy - a multicenter randomized controlled clinical trial. Clin Oral Implants Res. 2014;25(2):185-93. DOI: 10.1111/clr.12098.

33. Arduino PG, Tirone F, Schiorlin E, Esposito M. Single preoperative dose of prophylactic amoxicillin versus a 2-day postoperative course in dental implant surgery: A two-centre randomised controlled trial. Eur J Oral Implantol. 2015;8(2):143-9.

34. Esposito M, Grusovin MG, Talati M, Coulthard P, Oliver R, Worthington HV. Interventions for replacing missing teeth: antibiotics at dental implant placement to prevent complications. Cochrane Database Syst Rev. 2008;(3):CD004152. DOI: 10.1002/14651858.CD004152.pub2.

35. Abu-Ta'a M, Quirynen M, Teughels W, van Steenberghe D. Asepsis during periodontal surgery involving oral implants and the usefulness of peri-operative antibiotics: a prospective, randomized, controlled clinical trial. J Clin Periodontol. 2008;35(1):58-63. DOI: 10.1111/j.1600-051X.2007.01162.x.

36. Camps-Font O, Figueiredo R, Valmaseda-Castellon E, Gay-Escoda C. Postoperative Infections After Dental Implant Placement: Prevalence, Clinical Features, and Treatment. Implant Dent. 2015;24(6):713-9. DOI: 10.1097/ID.0000000000000325.

37. Hartshorne J. Do surgical prophylactic antibiotics reduce postoperative infection and early dental implant failure? Int Dent. 2014;4(5):6-11. DOI: 10.10.1111/joor.12211.

38. Sánchez-Martín G, del Río-Highsmith J. Protocolos antibióticos en Odontología. J Am Dent Assoc. 2009;4:289-96.

39. Testori T, Drago L, Wallace SS, Capelli M, Galli F, Zuffetti F, et al. Prevention and treatment of postoperative infections after sinus elevation surgery: clinical consensus and recommendations. Int J Dent. 2012;2012:365809. DOI: 10.1155/2012/365809.
40. Classen DC, Evans RS, Pestotnik SL, Horn SD, Menlove RL, Burke JP. The timing of prophylactic administration of antibiotics and the risk of surgical-wound infection. N Engl J Med. 1992;326(5):281-6. DOI: 10.1056/NEJM199201303260501.

41. Maureci R, Campisi G, Matranga D, Maureci N, Pizzo G, Melilli D. The Role of Antibiotic Prophylaxis in Reducing Bacterial Contamination of Autologous Bone Graft Collected from Implant Site. Biomed Res Int. 2017;2017:2175019. DOI: 10.1155/2017/2175019.

42. Brennan MT, Sasser HC, Fox PC, Lockhart PB. Statistical analysis used in "A prospective placebo-controlled double-blind trial of antibiotic prophylaxis in intraoral bone grafting procedures: a pliot study». Oral Surg Oral Med Oral Pathol Oral Radiol Endod. 2004;97(6):664; author reply 664-5.

43. Lambrecht J. Profilaxis y terapia antibióticas en cirugía oral: análisis de la literatura. Quintessence. 2008;21(7):405-13.

44. Piñeiro A, Tomás I, Blanco J, Alvarez M, Seoane J, Diz P. Bacteraemia following dental implants' placement. Clin Oral Implants Res. 2010;21(9):913-8. DOI: 10.1111/j.1600-0501.2010.01928.x

45. Wilson W, Taubert KA, Gewitz M, Lockhart PB, Baddour LM, Levison $\mathrm{M}$, et al. Prevention of infective endocarditis. Guidelines from the American Heart Association. A Guideline From the American Heart Association. Rheumatic fever, endocarditis, and Kawasaki Disease Committee, Council on Cardiovascular Disease in the Young, and the C. Circulation. 2007;116(15):1736-54. DOI: 10.1161/CIRCULATIONAHA.106.183095.

46. Lockhart PB, Brennan MT, Kent ML, Norton HJ, Weinrib DA. Impact of amoxicillin prophylaxis on the incidence, nature, and duration of bacteremia in children after intubation and dental procedures. Circulation. 2004;109(23):2878-84. DOI: 10.1161/01.CIR.0000129303.90488.29.

47. Roberts GJ, Radford P, Holt R. Prophylaxis of dental bacteraemia with oral amoxycillin in children. Br Dent J. 1987;162(5):179-82.

48. Starkebaum M, Durack D, Beeson P. The «incubation period» of subacute bacterial endocarditis. Yale J Biol Med. 1977;50(1):49-58.

49. Selton-Suty C, Celard M, Le Moing V, Doco-Lecompte T, Chirouze C, Iung B, et al. Preeminence of Staphylococcus aureus in infective endocarditis: a 1-year population-based survey. Clin Infect Dis. 2012;54(9):1230-9. DOI: 10.1093/cid/cis199.

50. Duval X, Delahaye F, Alla F, Tattevin P, Obadia JF, Le Moing V, et al. Temporal trends in infective endocarditis in the context of prophylaxis guideline modifications: three successive population-based surveys. J Am Coll Cardiol. 2012;59(22):1968-76. DOI: 10.1016/j.jacc.2012.02.029.

51. Dajani AS, Taubert KA, Wilson W, Bolger AF, Bayer A, Ferrieri P, et al. Prevention of bacterial endocarditis: recommendations by the American Heart Association. J Am Dent Assoc. 1997;128(8):1142-51.

52. Laskin DM, Dent CD, Morris HF, Ochi S, Olson JW. The influence of preoperative antibiotics on success of endosseous implants at 36 months. Ann Periodontol. 2000;5(1):166-74. DOI: 10.1902/ annals.2000.5.1.166.

53. Siddiqi A, Morkel JA, Zafar S. Antibiotic prophylaxis in third molar surgery: A randomized double-blind placebo-controlled clinical trial using split-mouth technique. Int J Oral Maxillofac Surg. 2010;39(2):107-14. DOI: 10.1016/j.ijom.2009.12.014.

54. Hill M. No benefit from prophylactic antibiotics in third molar surgery. Evid Based Dent. 2005;6(1):10. DOI: 10.1038/sj. ebd.6400307.

55. Sancho-Puchades M, Herraez-Vilas JM, Berini-Aytes L, GayEscoda C. Antibiotic prophylaxis to prevent local infection in Oral Surgery: use or abuse? Med Oral Patol Oral Cir Bucal. 2009;14(1):E28-33.

56. Danda AK, Ravi P. Effectiveness of postoperative antibiotics in orthognathic surgery: a meta-analysis. J Oral Maxillofac Surg. 2011;69(10):2650-6. DOI: 10.1016/j.joms.2011.02.060.

57. Solensky R. Hypersensitivity reactions to beta-lactam antibiotics. Clin Rev Allergy Immunol. 2003;24(3):201-20.

58. Kaur SP, Rao R, Nanda S. Amoxicillin: a broad spectrum antibiotic. Int J Pharm Pharm Sci. 2011;3(3):30-7. 
59. Khoury SB, Thomas L, Walters JD, Sheridan JF, Leblebicioglu B. Early wound healing following one-stage dental implant placement with and without antibiotic prophylaxis: a pilot study. J Periodontol. 2008;79(10):1904-12. DOI: 10.1902/jop.2008.070670.

60. Escalante MG, Eubank TD, Leblebicioglu B, Walters JD. Comparison of Azithromycin and Amoxicillin Before Dental Implant Placement: An Exploratory Study of Bioavailability and Resolution of Postoperative Inflammation. J Periodontol. 2015;86(11):1190-200. DOI: 10.1902/jop.2015.150024.

61. Larsson Wexell C, Ryberg H, Sjoberg Andersson WA, Blomqvist S, Colin P, Van Bocxlaer J, et al. Antimicrobial Effect of a Single Dose of Amoxicillin on the Oral Microbiota. Clin Implant Dent Relat Res. 2016;18(4):699-706. DOI: 10.1111/cid.12357.

62. Aravena PC, Oyarzun CP, Arias MF, Monardes H, Jerez A, Benso B. Single-Dose Bioavailability for Prophylactic Coverage in Patients Undergoing Dental Implant Surgery. Int J Oral Maxillofac Implants. 2018;33(2):419-24. DOI: 10.11607/jomi.5943.

63. Patel J, Cockerill FI, Bradford P, Eliopoulos G, Hindler J, Jenkins S, et al. M07-A9: Methods for Dilution Antimicrobial Susceptibility Tests for Bacteria That Grow Aerobically; Approved Standard - Tenth Edition; 2015.

64. Vincent J, Abraham E, Kochanek P, Moore F, Fink M. Textbook of critical care. $6^{\text {th }}$ editio. Philadelphia: Elsevier Health Sciences; 2011.
65. Blondeau JM. New concepts in antimicrobial susceptibility testing: the mutant prevention concentration and mutant selection window approach. Vet Dermatol. 2009;20(5-6):383-96. DOI: 10.1111/j.1365-3164.2009.00856.x.

66. Surapaneni H, Yalamanchili PS, Basha MH, Potluri S, Elisetti N, Kiran Kumar MV. Antibiotics in dental implants: A review of literature. J Pharm Bioallied Sci. 2016;8:S28-31. DOI: 10.4103/0975-7406.191961.

67. Dovi J, He L, DiPietro L. Accelerated wound closure in neutrophil-depleted mice. J Leukoc Biol. 2003;73(4):448-55.

68. Esposito M, Hirsch JM, Lekholm U, Thomsen P. Biological factors contributing to failures of osseointegrated oral implants. (II). Etiopathogenesis. Eur J Oral Sci. 1998;106(3):721-64.

69. Hwang D, Wang H. Medical contraindications to implant therapy: Part II: Relative contraindications. Implant Dent. 2007;16(1):13-23. DOI: 10.1097/ID.0b013e31803276c8.

70. Bressan E, Tessarolo F, Sbricoli L, Caola I, Nollo G, Di Fiore A. Effect of chlorhexidine in preventing plaque biofilm on healing abutment: a crossover controlled study. Implant Dent. 2014;23(1):64-8. DOI: 10.1097/ID.0000000000000018.

71. Resnik RR, Misch CE. Farmacología en Implantología dental. En: Misch CE, editor. Implantol. Contemp. $3^{\circ}$, Barcelona: Elsevier Mosby; 2009. p. 467-89. 